\title{
Avaliação de Revestimentos de Liga de Níquel 625 Depositados pelo Processo Eletroescória
}

\author{
Soraia Simões Sandes ${ }^{1}$, Camila Pereira Alvarães ${ }^{1}$, Matheus Campolina Mendes ${ }^{1}$, Leonardo Sales de Araújo², \\ Luis Felipe Guimarães de Souza ${ }^{1}$, Jorge Carlos Ferreira Jorge ${ }^{1}$
}

1 Centro Federal de Educação Tecnológica - CEFET, Diretoria de Pesquisa e Pós-graduação - DIPPG, Rio de Janeiro, RJ, Brasil.

2 Universidade Federal do Rio de Janeiro - UFRJ, Instituto Alberto Luiz Coimbra de Pós-graduação e Pesquisa de Engenharia COPPE, Rio de Janeiro, RJ, Brasil.

Recebido: 16 Ago., 2016

Aceito: 09 Nov., 2016

E-mails: soraia.sandes@hotmail.com (SSS), camila.alvaraes@gmail.com (CPA), campolinamendes@gmail.com (MCM), Leonardo.sales@gmail.com (LSA), Ifgs59@gmail.com (LFGS), jorgecfjorge@gmail.com (JCFJ)
Resumo: A aplicação de revestimentos de ligas de níquel é comumente realizada na indústria de petróleo e gás para aumento da vida de equipamentos em ambientes agressivos, dado que este procedimento melhora a resistência à corrosão, sem um aumento significativo do custo de produção quando comparado com equipamentos maciços fabricados com outras ligas. Normalmente, as juntas são soldadas pelos processos de eletrodos revestidos, MIG/MAG ou TIG. Neste aspecto, o processo eletroescória realiza deposições com elevada energia de soldagem e baixa diluição, podendo ser uma opção interessante, uma vez que proporciona uma elevada produtividade, permitindo a soldagem com aplicação de apenas uma camada. O presente trabalho avalia propriedades mecânicas, microestruturais e de corrosão de revestimentos de liga de níquel 625 depositada em aço carbono ASTM A516 Grau 70 pelo processo eletroescória, tanto na condição como soldado quanto tratado termicamente. A deposição foi realizada com uma e duas camadas sobre chapas de dimensão 50×400x400 mm, na posição plana e energia de soldagem média de 11,7 kJ/mm. Após a soldagem realizou-se tratamento térmico a $620^{\circ} \mathrm{C}$ por 10 horas, sendo esta condição comparada ao estado de como soldado. Os ensaios de dobramento não indicaram evidências de defeitos. A avaliação microestrutural realizada por microscopia ótica (MO), eletrônica de varredura (MEV) e eletrônica de transmissão (MET) mostrou uma microestrutura austenítica para o depósito com pequena fração volumétrica de fases secundárias e o tratamento térmico de alívio de tensões não promoveu mudanças significativas nas propriedades. Na região de grãos grosseiros da zona termicamente afetada (RGGZTA) foi observada a ocorrência de ferrita pró-eutetóide, perlita e bainita para o depósito com 1 camada e basicamente ferrita e perlita refinadas para o deposito com 2 passes devido às baixas taxas de resfriamento. Adicionalmente, não foi verificada evidência de zona parcialmente diluída (ZPD). Os ensaios de corrosão realizados de acordo com a norma ASTM G-48 em amostras retiradas a partir da camada superior do depósito foram considerados satisfatórios, uma vez que não mostraram evidências de pites e a perda de massa foi pequena.

Palavras-chave: Revestimento; Liga de níquel 625; Eletroescória.

\section{Evaluation of the 625 Nickel Alloy Weld Overlays Deposited by Electroslag Process}

Abstract: Nickel base alloy weld overlays are commonly used in oil \& gas industry to extend
the life of equipments under aggressive corrosion environments, since the overlays improve
the corrosion resistance without a significant increase the cost of manufacture when
compared to massive equipment. Usually, the joints are welded by SMAW, GMAW or GTAW
processes. In this respect, the electro slag welding process (ESW), which promotes high heat
inputs and low dilution welds, can be an interesting option for this application as it provides a
high productivity, once only one layer is necessary. The present work evaluates mechanical,
microstructural and corrosion properties of an Alloy 625 weld overlay deposited on ASTM
A516 Grade 70 carbon steel by ESW process. The deposition was done with 1 and 2 layers
in plates of dimension 50x400x400 mm, in the flat position and an average energy welding
11.7 kJ/mm. After welding, a post weld heat treatment at $620^{\circ} \mathrm{C}$ for 10 hours was performed,
this condition being compared with the as welded condition. Bending tests showed no evidences
of cracks. Microstructural evaluation performed using both optical (OM), scanning (SEM) and
transmission electron microscopy (TEM) techniques showed an austenitic microstructure of
the weld deposit with low proportion of secondary phases for all conditions and the post
welding heat treatment did not promote significant changes on the mechanical properties.
At the coarse grain heat affected zone (CGHAZ) the occurrence of proeutectoid ferrite, pearlite
Este é um artigo publicado em acesso aberto (Open Access) sob a licença Creative Commons Attribution Non-Commercial, que permite uso, distribuição e reproduçãa em qualquer meio, sem restriçōes desde que sem fins comerciais e que 0 trabalho original seja corretamente citado. 
and bainite were found for 1 layer deposit and refined pearlite and ferrite for 2 layer deposits were the microstructural constituents observed due to the low cooling rate. In addition, no evidences of partially diluted zones (PDZ) were verified. Corrosion tests conducted on samples removed from top layer of weld overlay cladding deposit according to ASTM G 48 Method A Standard were considered satisfactory once no evidences of pittings were verified and the loss of mass was very reduced.

Key-words: Overlay; Nickel alloy 625; Eletroslag.

\section{Introdução}

Uma alternativa para substituição de materiais de custo elevado em ambientes agressivos é o revestimento de aço C-Mn com superligas de níquel [1-5]. Este procedimento é atrativo, visto contribuir para a melhoria da resistência à corrosão, sem aumento do custo de fabricação do componente, quando comparado com o equipamento totalmente fabricado em material especial [3,6].

As ligas à base de níquel são amplamente utilizadas em diversas áreas industriais, tais como química, petroquímica, reatores nucleares, bélica, aeroespacial, dispositivos de processamento de alimentos e instalações de produção de aço [7-9], devido a uma associação de alta resistência mecânica, boa resistência à corrosão e boa soldabilidade [1]. O desempenho destas ligas está associado com a estrutura cúbica de face centrada da matriz, que pode ser endurecida por solução sólida ou por precipitação de compostos intermetálicos.

O revestimento por soldagem pode ser obtido por vários processos, tais como eletrodos revestidos, MIG/MAG, arame tubular, TIG, explosão e plasma [2,5,7,9-18]. Em alguns casos, o tratamento térmico pós-soldagem (TTPS) é por vezes recomendado para aliviar tensões residuais, reduzir o teor de hidrogênio, ou homogeneizar a microestrutura [1,9,19]. Neste aspecto, Kahar e Baba Pai [11] comentam que os dois processos mais produtivos para revestimento de grandes componentes que estão sujeitos à corrosão ou desgaste são os processos a arco submerso e eletroescória, ambos com fita. Embora o processo a arco submerso (SAS) seja mais utilizado, quando se requer maior produtividade e menores taxas de diluição a soldagem pelo processo eletroescória (SEE) é recomendada. Neste aspecto, Bedi et al. [10] afirmam que o processo SEE é mais eficiente devido às suas propriedades únicas, como alta taxa de deposição, baixa diluição e depósitos de alta qualidade. O processo eletroescória com fita tem sido muito utilizado no revestimento de equipamentos nas indústrias química, petroquímica e nuclear [19].

O presente trabalho avalia o comportamento das propriedades mecânicas, microestruturais e de corrosão de revestimentos de liga de níquel 625 depositados pelo processo eletroescória com fita sobre o aço carbono ASTM A 516 Grau 70, com 1 e 2 camadas para avaliar algum possível efeito do reaquecimento nas características do revestimento, tanto na condição de como soldado quanto após tratamento térmico.

\section{Materiais e Métodos}

\subsection{Materiais}

Chapas de aço carbono ASTM A516 Gr. 70 de dimensão 50x400x400 mm foram utilizadas como material de substrato. Uma fita com as dimensões 0,5 mm de espessura e $60 \mathrm{~mm}$ de largura da classe AWS 5.14 EQ-NiCrMo-3 foi usada para produzir o revestimento com a liga de níquel 625. A Tabela 1 mostra a composição química dos materiais, segundo informações dos fabricantes.

Tabela 1. Composição química dos materiais (\% Peso).

\begin{tabular}{lcccccccc}
\hline \multicolumn{1}{c}{ Elemento, \% peso } & $\mathbf{C}$ & $\mathbf{S i}$ & $\mathbf{M n}$ & $\mathbf{N i}$ & $\mathbf{C r}$ & $\mathbf{M o}$ & $\mathbf{N b}$ & $\mathbf{F e}$ \\
Aço ASTM A516 Gr.70 & 0,15 & 0,20 & 0,95 & 0,01 & 0,02 & 0,01 & - & Bal. \\
Liga de níquel 625 & 0,03 & 0,40 & 0,30 & Bal. & 19,5 & 8,0 & 2,8 & 3,00 \\
\hline
\end{tabular}




\subsection{Soldagem}

Os revestimentos foram depositados pelo processo eletroescória com fita com 2 procedimentos diferentes. O primeiro depósito foi realizado em quatro passes em camada única na posição plana de soldagem, enquanto o segundo depósito foi realizado em duas camadas, sendo a primeira realizada com as mesmas condições do primeiro depósito e a segunda camada em 2 passes. Utilizaram-se os parâmetros de soldagem mostrados na Tabela 2.

Após a soldagem, foram realizados tratamentos térmicos consistindo de aquecimento a $620^{\circ} \mathrm{C}$ por 10 horas, seguido de resfriamento ao ar, sendo esta condição comparada à condição de como soldado do metal de solda.

Tabela 2. Parâmetros de soldagem utilizados.

\begin{tabular}{ccc}
$\begin{array}{c}\text { Corrente } \\
(\mathbf{A})\end{array}$ & $\begin{array}{c}\text { Tensão } \\
\text { (Volts) }\end{array}$ & $\begin{array}{c}\text { Energia de soldagem } \\
(\mathbf{k J} / \mathbf{m m})\end{array}$ \\
$1150-1250$ & $24-26$ & 11,7 \\
\hline
\end{tabular}

\subsection{Ensaios mecânicos}

Foram realizados ensaios de microdureza Vickers com aplicação de carga de 500 gf, consistindo de varredura de dureza perpendicularmente à linha de fusão, com espaçamento das impressões de 0,25 mm, abrangendo o metal base, interface e metal depositado. Foram ainda realizados ensaios de dobramento de acordo com a norma ASME Seção IX [20].

\subsection{Ensaios metalográficos}

Realizou-se análise metalográfica, consistindo de macrografia, micrografia ótica (MO) e microscopia eletrônica de varredura (MEV) e análise por espectroscopia de energia dispersiva (EDS) para caracterizar a microestrutura, identificação e quantificação de fases secundárias e ocorrência de zonas parcialmente diluídas (ZPD) próximas à linha de fusão. Para a análise quantitativa das fases secundárias, utilizou-se a técnica da contagem por pontos em grade metalográfica com 100 pontos na tela do MEV e aumento de 1.000 vezes. Foram contados aleatoriamente pelo menos 10 campos para cada condição, perfazendo um total de 1.000 pontos por amostra, localizados na posição relativa a $3 \mathrm{~mm}$ da linha de fusão. Para análise da precipitação de fases endurecedoras no revestimento foram retiradas amostras a cerca de $3 \mathrm{~mm}$ da linha de fusão para microscopia eletrônica de transmissão (MET).

A preparação das amostras consistiu da técnica convencional de lixamento e polimento, seguido de ataque químico eletrolítico de ácido oxálico a $10 \%$ e 6 volts para o metal de solda e ataque convencional de nital $2 \%$ para o metal base. Para a análise por MET, foram preparados discos com $3 \mathrm{~mm}$ de diâmetro e afinamento final da região de observação por meio eletrolítico (Tenupol 5) em solução de $9 \%$ de ácido perclórico e $91 \%$ de etanol, a $-20^{\circ} \mathrm{Ce} 30 \mathrm{~V}$.

De forma a se obter uma estimativa das fases formadas no revestimento foram realizados cálculos termodinâmicos usando software Thermocalc e a base de dados TTNI8. A composição química considerada foi a cerca de $3 \mathrm{~mm}$ da linha de fusão.

\subsection{Análise química}

Foi realizada análise química por espectrometria de emissão ótica em amostras do revestimento removidas a diferentes distâncias da linha de fusão, de forma a permitir uma análise da variação dos principais elementos de liga ao longo do revestimento, como o níquel e o cromo e ferro.

\subsection{Ensaios de corrosão}

Os ensaios de corrosão foram realizados de acordo com a norma ASTM G-48 [21], método A, tendo como critério de aceitação a norma NORSOK M 601 [22]. As amostras foram removidas do revestimento na posição relativa a $3 \mathrm{~mm}$ da linha de fusão. 


\section{Resultados e Discussão}

De acordo com Pachold [23], a preferência pela deposição de revestimentos pelo processo eletroescória, dentre outras razões, encontra suporte na possiblidade da execução do revestimento em uma única camada. No entanto, podem existir situações onde a operação de soldagem possa envolver passes adicionais, como por exemplo, em situações de reparos. Desta forma, o presente trabalho realizou uma análise com procedimentos diferentes, 1 e 2 camadas, para avaliar algum possível efeito do reaquecimento nas características do revestimento.

A Figura 1 mostra aspectos metalográficos do depósito realizado, onde é possível observar as regiões do revestimento, as quais também foram observadas em outros trabalhos técnicos $[3,16,24]$.

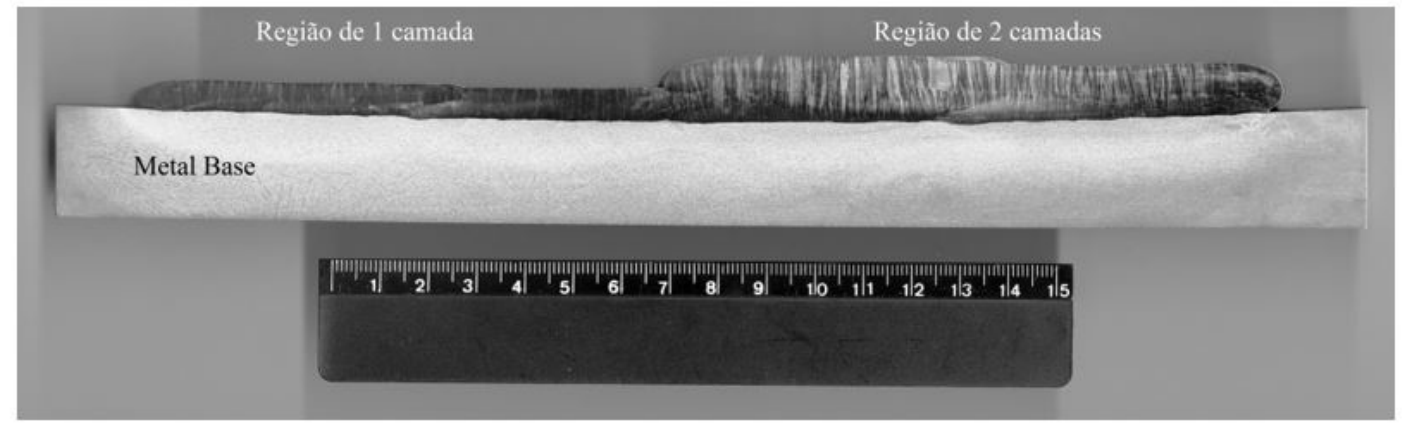

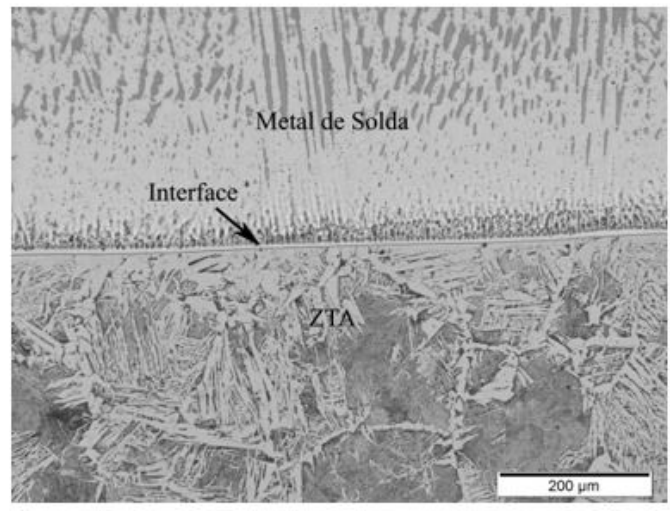

Região de 1 camada (MO)

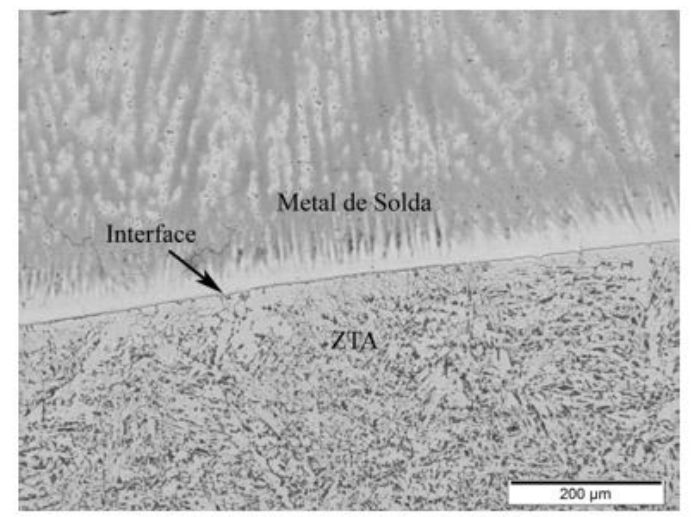

Região de 2 camadas (MO)

Figura 1. Aspectos metalográficos do revestimento.

A Figura 2 mostra que o teor de Fe diminui na medida em que se afasta da linha de fusão em direção ao revestimento. Pode-se observar que somente para a soldagem com 1 camada, o teor de Fe se situa em patamar inferior ao valor limite de $5 \%$ em distâncias inferiores ao limite de $3 \mathrm{~mm}$ de espessura de camada o qual é sugerido para o bom desempenho em corrosão do revestimento [5,16,25]. Este comportamento faz com que a soldagem com 2 camadas não seja adequada para o revestimento de equipamentos industriais.

No caso da soldagem em camada única, procedimento recomendado para a soldagem pelo processo eletroescória, a taxa de diluição de 4,0\%, calculada com o auxílio do software Auto CAD, pode ser considerada baixa quando comparada com outros processos [2,15,26,27]. Adicionalmente, não se observou evidências de defeitos ou desplacamento.

A reduzida taxa de diluição associada com a elevada energia de soldagem também contribuiu para evitar a ocorrência de zonas parcialmente diluídas (ZPD) ao longo da interface (Figura 1), normalmente observadas em depósitos por outros processos a arco elétrico [2,3,15,28], o que é um importante indicativo para o desempenho adequado do revestimento. Esta evidência também é suportada pelos ensaios de dureza (Figura 3), que apresentam resultados muito inferiores a $350-400 \mathrm{HV}$, valores que estão geralmente associados à ocorrência de ZPD [2,3,15,28]. 

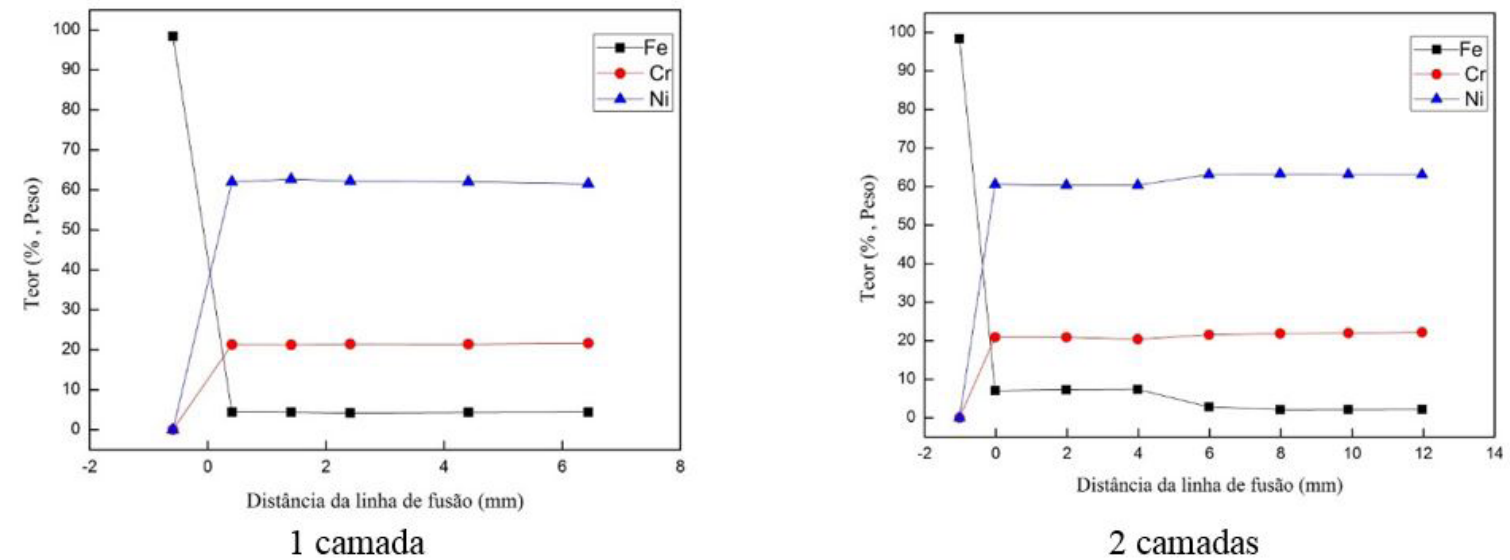

Figura 2. Variação do teor dos principais elementos a partir da linha de fusão.

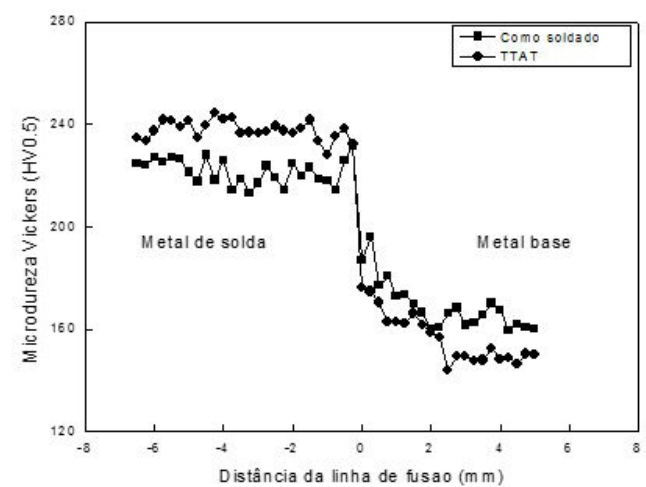

1 camada

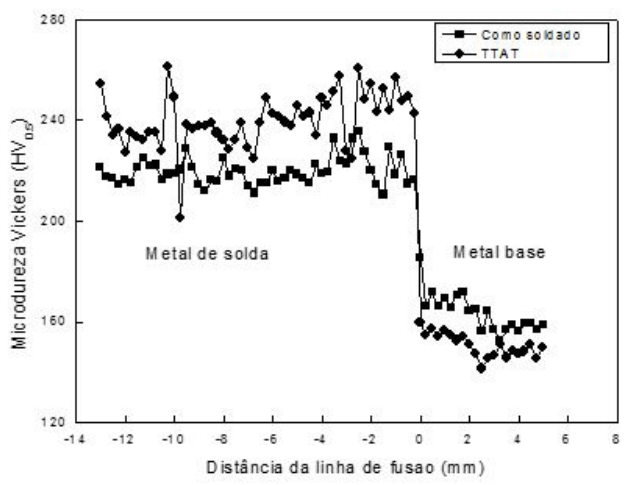

2 camadas

Figura 3. Resultados dos ensaios de microdureza.

Em relação ao metal de solda, a análise por microscopia ótica (Figura 1) mostrou uma microestrutura totalmente austenítica, com uma forma de solidificação usual para revestimentos com a liga de níquel 625 [6] e apresentando um elevado nível de segregação e ocorrência de fases secundárias (Figura 4). Neste aspecto, a Tabela 3, que mostra o valor do coeficiente de distribuição (k), contribui para o entendimento, já reportado pela literatura [4,14,29-32] sobre a segregação de elementos como o Mo e Nb para a região interdendrítica, enriquecendo essa região e, consequentemente, empobrecendo o centro das dendritas desses elementos e os enriquecendo em elementos como o Ni. De fato, nota-se a tendência de uma leve segregação dos elementos $\mathrm{Ni}$ e $\mathrm{Cr}$ para o sólido $(k>1)$ e de um potente efeito de segregação para as regiões interdendríticas do Mo e, principalmente do $\mathrm{Nb}$, sendo este fato computado como responsável para a formação de fases secundárias em revestimentos com a liga de níquel $625[4,32]$.

Tabela 3. Coeficiente de distribuição k: segregação de elementos.

\begin{tabular}{cccc}
\hline Posição & Centro da Dendrita $\left(\mathbf{C}_{\mathbf{s}}\right)$ & Região Interdendrítica $\left(\mathbf{C}_{\mathbf{i}}\right)$ & $\mathbf{K}$ \\
$\mathrm{Cr}$ & 22,58 & 19,99 & 1,13 \\
$\mathrm{Ni}$ & 61,63 & 55,49 & 1,11 \\
$\mathrm{Mo}$ & 8,85 & 12,61 & 0,70 \\
$\mathrm{Nb}$ & 1,90 & 7,71 & 0,25 \\
\hline
\end{tabular}




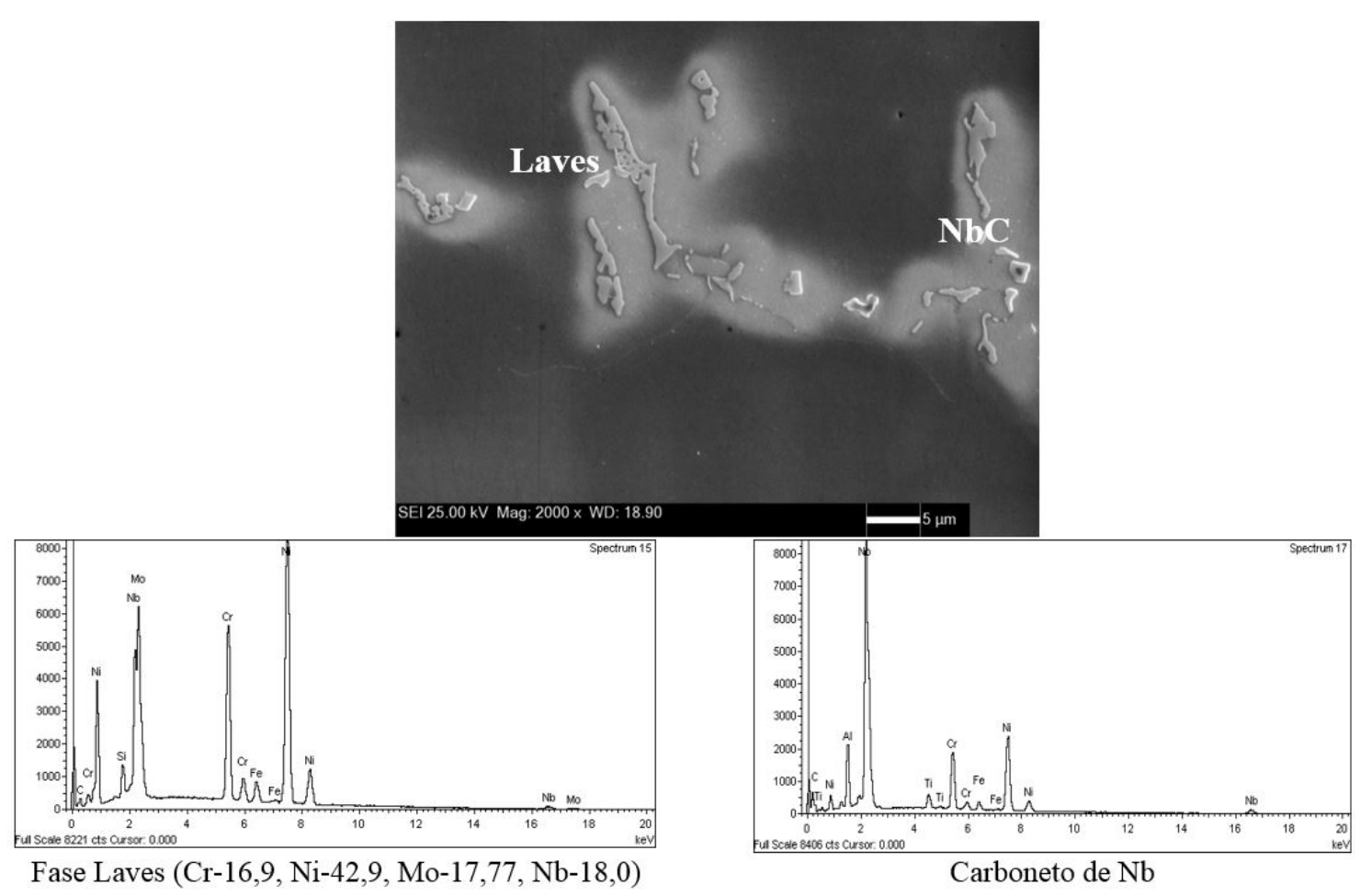

Figura 4. Ocorrência de segregação e fases secundárias no metal de solda (MEV). Ataque: Ácido oxálico.

De acordo com Cieslak et al. [33], as ligas sem Nb solidificam com transformação simples $L \rightarrow \gamma$, sem qualquer reação eutética e apresentam uma faixa de temperaturas de solidificação relativamente estreita. Por outro lado, as ligas contendo Nb solidificam apresentando reação do tipo eutética e várias fases secundárias, tais como NbC e Laves. Du Pont et al. [19] afirmam que a seguinte sequência ocorre em ligas contendo Nb:

\section{1. $L \rightarrow L+\gamma \rightarrow$}

\section{2. $\mathrm{L}+\gamma+\mathrm{NbC} \rightarrow \mathrm{L}+\gamma+\mathrm{NbC}+\mathrm{M}_{6} \mathrm{C} \rightarrow$}

\section{3. $L+\gamma \mathrm{NbC}+\mathrm{M}_{6} \mathrm{C}+$ Laves $\rightarrow \gamma+\mathrm{NbC}+\mathrm{M}_{6} \mathrm{C}+$ Laves.}

Como suporte na previsão das fases presentes no revestimento, a Figura 5 mostra diagrama obtido por meio de cálculos termodinânicos com o uso do software Thermocalc e a base de dados TTNI8. Dado que a deposição e a solidificação do revestimento por eletroescória foi realizada em uma única etapa, um diagrama de solidificação foi construído, mostrando as fases formadas ao longo do resfriamento. Tal diagrama é fundamentado nas equações de Scheil [34]. Para a liga, a sequência de formação de fases previstas no diagrama é: $L \rightarrow L+\gamma \rightarrow L+\gamma+N b C+\rightarrow L+$ $\gamma+\mathrm{NbC}+$ Laves $\rightarrow \mathrm{L}+\gamma+\mathrm{NbC}+$ Laves $+\sigma \rightarrow \mathrm{L}+\gamma+\mathrm{NbC}+$ Laves $+\delta \rightarrow \mathrm{L}+\gamma+\mathrm{Laves}+\delta+\mathrm{M}_{6} \mathrm{C}+\sigma$. Cabe ressaltar que, durante a solidificação, há segregação de Mo e Nb para o líquido interdendrítico, enriquecendo localmente tais regiões e induzindo a formação de fases ricas nesses elementos como $\sigma$ e Laves. A Figura 6 mostra diagramas pseudo-binários com $\mathrm{Nb}$ e Mo, mostrando que a segregação desses elementos induz a formação de fases como Laves e $\sigma$.

Dado que um tratamento térmico por 10 horas a $620^{\circ} \mathrm{C}$ foi realizado após a deposição do revestimento, cálculos do equilíbrio de fases formadas nessa temperatura também foram realizados. Para essa temperatura, as precipitações das fases $\sigma$ e $\gamma^{\prime \prime}$ foram previstas. A fase $\gamma^{\prime \prime}$ é metaestável, de estrutura tetragonal de corpo centrado e, na liga de níquel 625 sua ocorrência pode induzir ganho de resistência.

Os resultados obtidos no presente trabalho estão em concordância com esta previsão, pois foi observada a presença de fases secundárias, Laves rica em Nb e carbonetos de $\mathrm{Nb}$ (Figura 4), com fração volumétrica inferior a $1,5 \%$ no metal de solda estudado. Estes resultados são consistentes com o relatado por diversos outros autores $[1,3,7,15,19,24,33,35-38]$. 


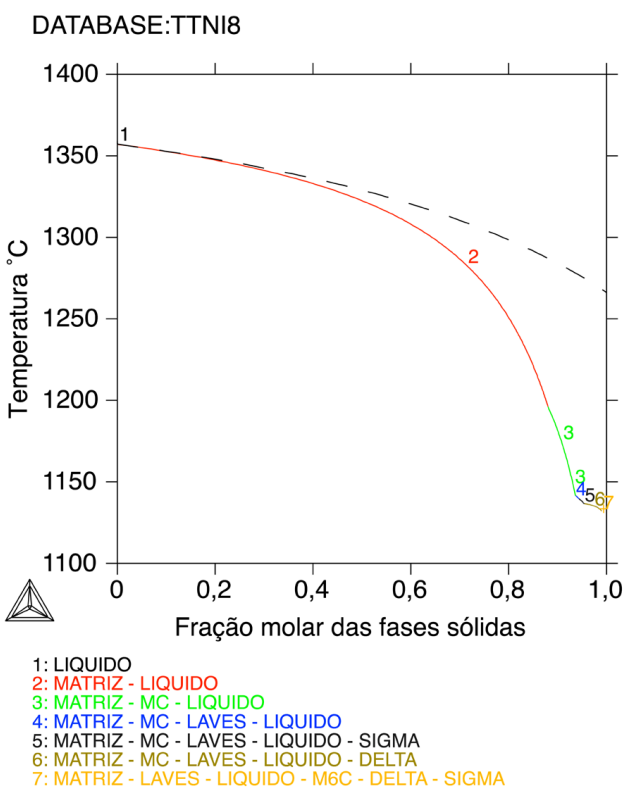

Figura 5. Diagrama de Scheil para os metais de solda.

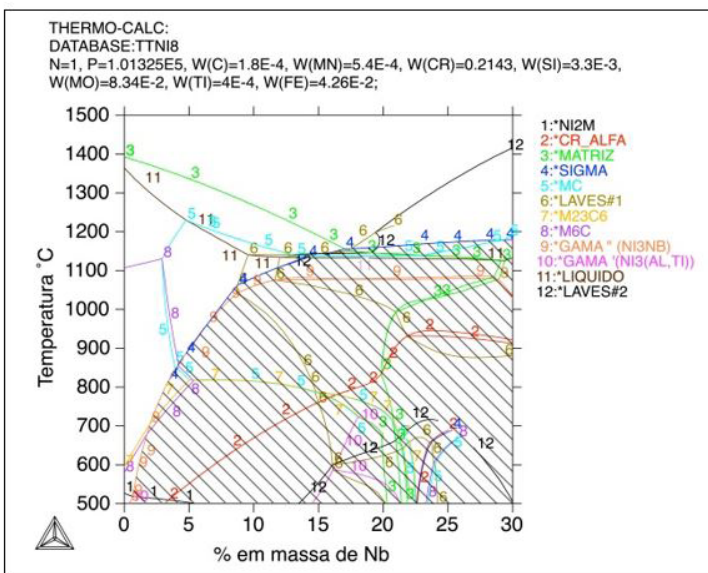

(a)

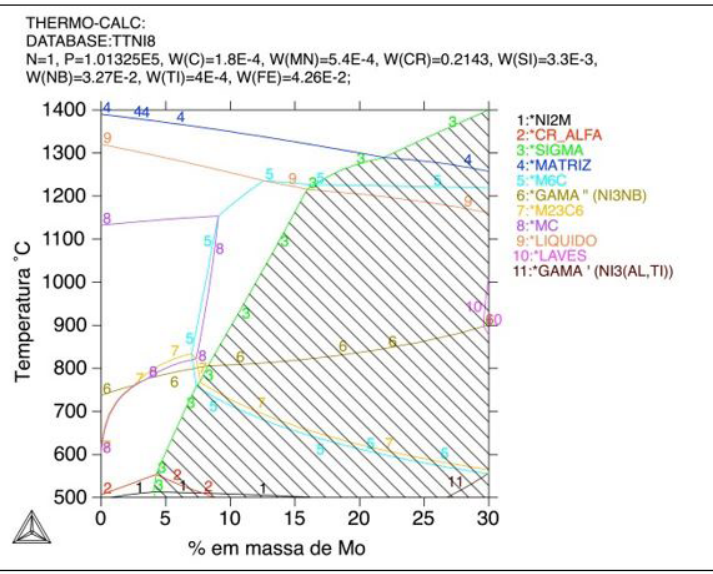

(b)

Figura 6. Diagrama pseudo-binário para o metal de solda com variações de: (a) Nb; (b) Mo. As regiões hachuradas mostram o campo de formação da fase $\sigma$.

Devido a elevada energia de soldagem aplicada, a microestrutura da região de grãos grosseiros da ZTA (RGGZTA) foi constituída de ferrita pró-eutetóide, perlita e bainita para o depósito com 1 camada. Já para o depósito com 2 camadas, a região do metal base próximo à interface, aqui identificada como região de grão grosseiro normalizada da ZTA (RGGNZTA), apresentou ferrita e perlita refinadas, como consequência do efeito de reaquecimento provocado pelo ciclo térmico desta segunda camada (Figura 7). Estas microestruturas com baixa dureza (Figura 3), também são de relevância, pois diferem da microestrutura martensítica de maior dureza normalmente encontrada nesta região quando se realiza o depósito por outros processos de soldagem $[15,17,28]$. No caso específico do presente trabalho, os maiores valores de dureza no metal base foram sempre inferiores a $250 \mathrm{HV}$, valor recomendado pela norma NACE M0175 [39] para a qualificação de procedimentos de soldagem.

O tratamento térmico de alívio de tensões (TTAT) é realizado algumas vezes para aliviar as tensões residuais, reduzir o teor de hidrogênio, homogeneizar a microestrutura ou manter os níveis dos valores de dureza dentro de 
determinados patamares $[19,24,26,40]$. Contudo, fases podem ser precipitadas durante a realização do tratamento térmico. A este respeito, Cortial et al. [1] estudando o efeito do TTAT na microestrutura e propriedades mecânicas de depósitos realizados com a liga de níquel 625, observaram um aumento da resistência mecânica e redução na ductilidade devido à precipitação da fase $\gamma^{\prime \prime}\left(\mathrm{Ni}_{3} \mathrm{Nb}\right)$ no espaço interdendrítico somente para temperaturas superiores a $750^{\circ} \mathrm{C}$. No entanto, em trabalhos recentes, Suave et al. [41,42] observaram o aparecimento desta fase para temperaturas de envelhecimento entre $550-750^{\circ} \mathrm{C}$. Conforme observado Figura 8 , a observação por MET confirma a precipitação de $\gamma^{\prime \prime}$ ao longo da matriz, na forma de precipitação fina, o que indica que o aumento da dureza no metal de solda após o TTAT (Figura 3) está associado com a precipitação desta fase, conforme verificado em outros estudos disponíveis [1,19,37,41,42].

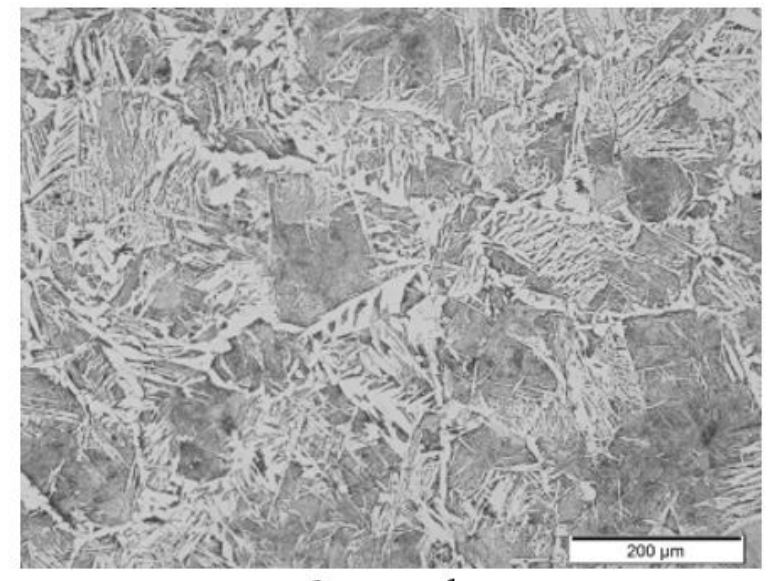

1 camada

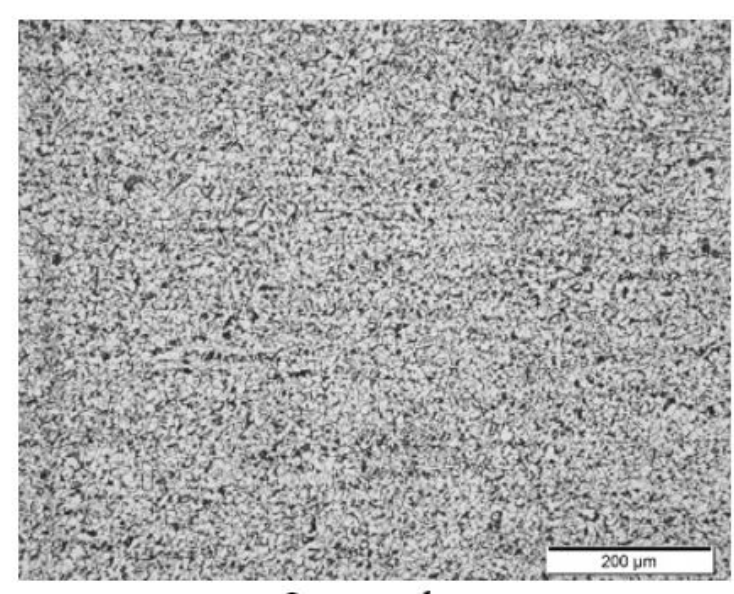

2 camadas

Figura 7. Microestrutura da RGGZTA para uma camada e RGGNZTA para duas camadas (MO). Ataque: nital $2 \%$.
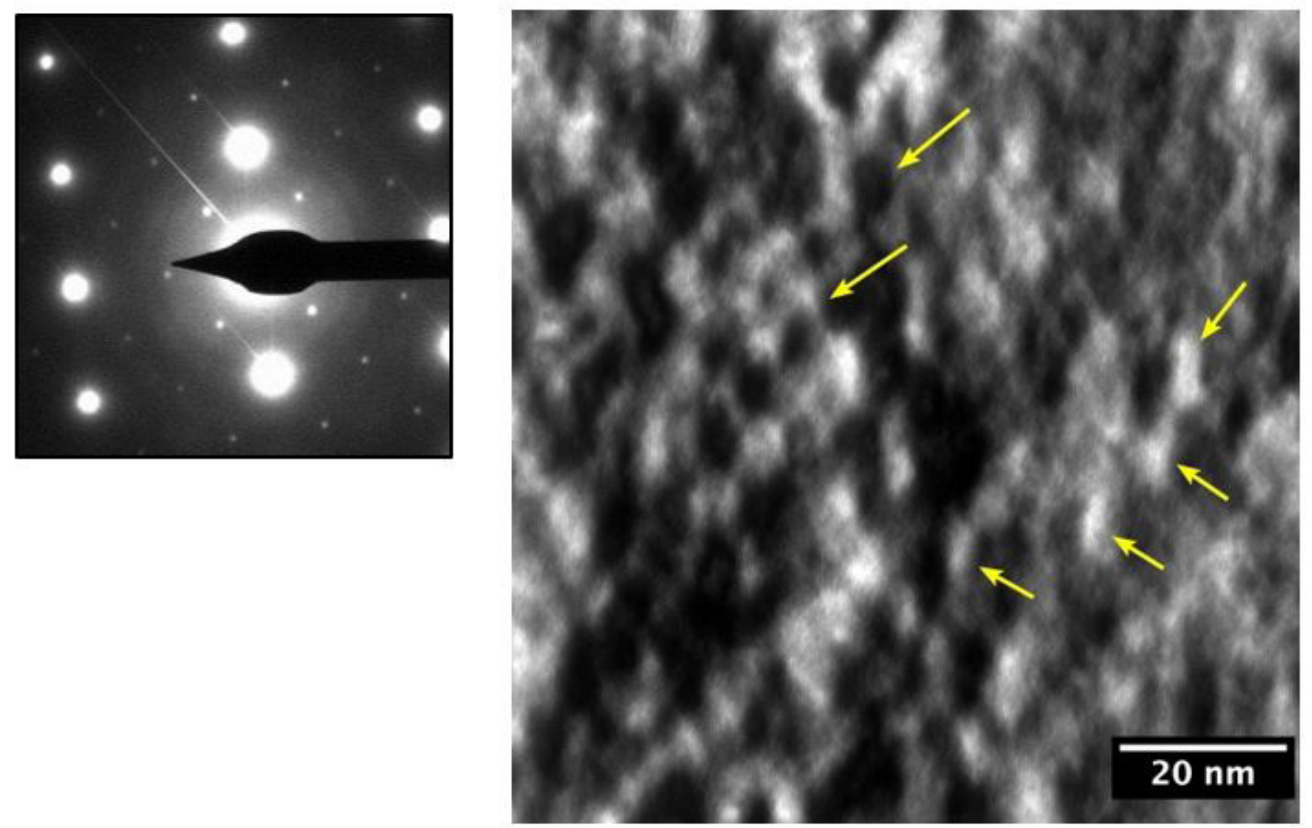

Figura 8. Ocorrência de fina precipitação de fase $\psi^{\prime \prime}$ após TTAT no metal de solda observado por MET. No canto superior esquerdo é apresentado o padrão de difração de elétrons, onde os pontos claros maiores referem-se à matriz e os menores aos precipitados $\gamma^{\prime \prime}$. As setas amarelas indicam os precipitados $\gamma^{\prime \prime}$, finos em sua ocorrência e com contornos difusos, em função de sua deformação de coerência. 
Na ZTA, o TTAT promoveu o revenido da microestrutura com a consequente redução na dureza (Figura 3).

De uma forma geral, o TTAT realizado a $620^{\circ} \mathrm{C}$ não causou alterações significativas nas propriedades mecânicas, pois embora tenha contribuído para pequenas mudanças nos resultados dos ensaios de dureza (Figura 3), não se observou incidência de defeitos nos ensaios de dobramento (Figura 9).

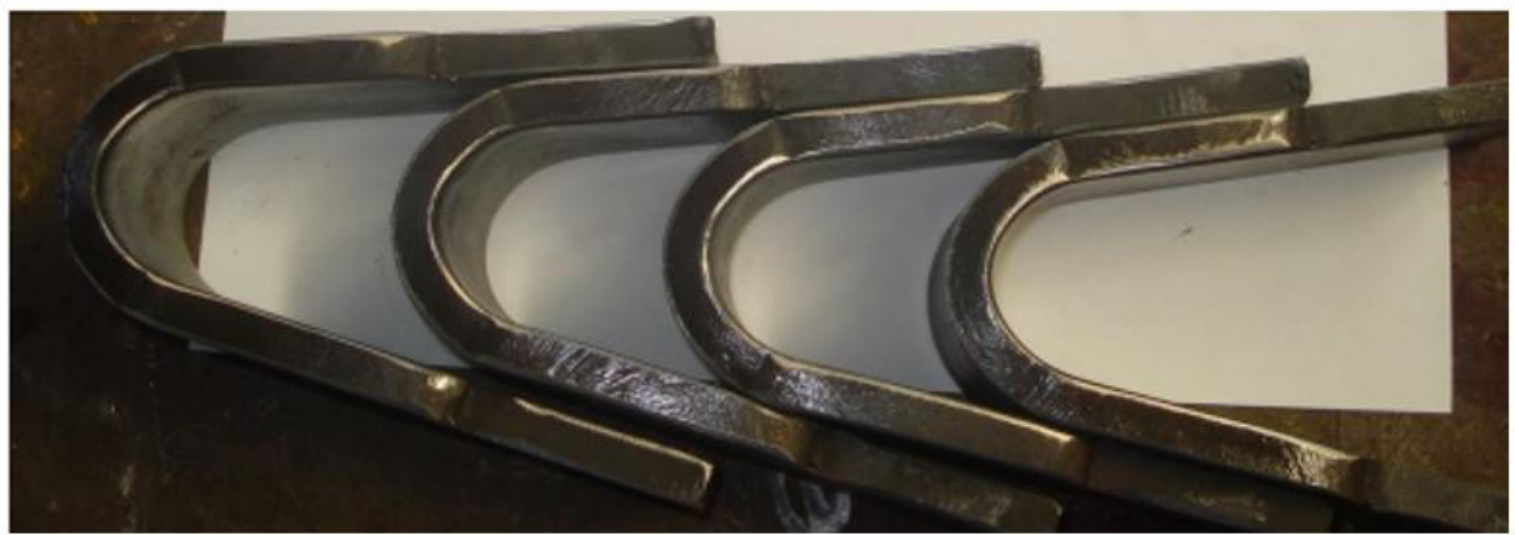

Figura 9. Aspecto dos corpos-de-prova de dobramento após o ensaio.

Com base nos resultados obtidos, avalia-se que o processo eletroescória em camada única possa ser uma alternativa atrativa na deposição de revestimentos de liga de níquel 625 sobre aços carbono, uma vez que permite a obtenção de revestimentos sem incidência de defeitos e com propriedades mecânicas e microestruturais adequadas, apresentando, inclusive, menor risco à ocorrência de problemas na interface devido as elevadas energias de soldagem aplicadas. Foi também possível verificar somente pequenas alterações devido à realização do TTAT, o que é mais um fator de importância devido à estabilidade do depósito, quando se fizer necessária a execução de reparos. Assim, a opção pela deposição em passe único apresenta vantagens por fazer uma melhoria na produtividade nas operações de deposição.

No sentido de confirmar a possibilidade de utilização deste processo na deposição de revestimentos com qualidade e elevada produtividade, com apenas uma única camada, foram realizados ensaios de corrosão de acordo com a norma exigida para qualificação de procedimentos [21]. A Tabela 4 mostra os resultados dos ensaios, evidenciando resultados satisfatórios e com perda de massa inferior a observada em outros processos [15].

Tabela 4. Resultados dos ensaios de corrosão.

\begin{tabular}{ccc}
\hline Condição & Pites & Perda de $\mathbf{m a s s a}\left(\mathbf{g} / \mathbf{m}^{2}\right)$ \\
Como soldado & Não & 0,0 \\
TTAT & Não & 0,17 \\
Requisito Norsok [22] & Não & $<4,00$ \\
\hline
\end{tabular}

\section{Conclusões}

Do exposto no transcurso do presente trabalho, pode-se concluir:

a) Revestimentos realizados pelo processo eletroescória com 1 camada apresentam boas propriedades mecânicas e de corrosão, apesar da ocorrência de fases secundárias no metal de solda;

b)O TTAT não promove mudanças significativas nas propriedades dos revestimentos;

c) A deposição pelo processo eletroescória com camada única pode ser uma alternativa interessante para revestimentos de liga 625 sobre aço carbono, devido às boas propriedades mecânicas e de corrosão, baixa taxa de diluição e elevada produtividade. 


\section{Agradecimentos}

Os autores agradecem às seguintes Instituições pelo apoio prestado na execução do presente trabalho: CEFET/RJ, PETROBRAS, UFRJ, CNPq e FINEP. Estes também são devidos ao Núcleo Multiusuário de Microscopia da COPPE/UFRJ pelas imagens de microscopia de transmissão.

\section{Referências}

[1] Cortial F, Corrieu JM, Vernot-Loier C. Heat treatments of weld alloy 625: Influence on the microstructure, mechanical properties and corrosion resistance. In: Proceedings of the Speralloys 718, 625, 706 and Various Derivatives; 1994 May 18-20; Nantes, France. Nantes: The Minerals, Metals \& Materials Society; 1994. p. 859-870.

[2] Kejelin NZ. Soldagem de revestimento de aços comuns C-Mn com superliga a base de níquel Inconel 625 [tese de doutorado]. Florianópolis: Universidade Federal de Santa Catarina; 2012.

[3] Silva CC, Afonso CRM, Ramirez AJ, Motta MF, Miranda HC, Farias JP. Aspectos metalúrgicos de revestimentos dissimilares com a superliga a base de níquel Inconel 625. Soldagem \& Inspeção. 2012;17(3):251-263. http://dx.doi.org/10.1590/ S0104-92242012000300009.

[4] Silva CC, Miranda HC, Motta MF, Farias JP, Afonso CRM, Ramirez AJ. New insight on the solidification path of an alloy 625 weld overlay. Journal of Materials Research and Technology. 2013;2(3):228-237. http://dx.doi.org/10.1016/j.jmrt.2013.02.008.

[5] Elango P, Balaguru S. Welding parameters for Inconel 625 overlay on carbon steel using GMAW. Indian Journal of Science and Technology. 2015;31(11):1-5.

[6] Kim JS, Park YI, Lee HW. Effects of heat input on the pitting resistance of Inconel 625 welds by overlay welding. Metals and Materials International. 2015;21(2):350-355. http://dx.doi. org/10.1007/s12540-015-4245-9.

[7] Antoszczyszyn TJ, Paes RMG, Oliveira ASCM, Scheid A. Impact of dilution on the microstructure and properties of Ni-Based 625 alloy coatings. Soldagem \& Inspeção. 2014;19(2):134-144. http://dx.doi.org/10.1590/0104-9224/SI1902.05.

[8] LiS, Wei Q, Shi Y, Zhu Z, Zhang D. Microstructure characteristics of Inconel 625 superalloy manufactured by selective laser melting. Journal of Materials Science and Technology. 2015;31(9):946952. http://dx.doi.org/10.1016/j.jmst.2014.09.020.

[9] Devletian JH, Gao YP, Zhao QH. Strip cladding of main propeller shafting with Ni alloy 625 by electroslap surfacing. In: Proceedings of the Ship Production Symposium; 1992 Sept 2-4; New Orleans, USA. New Orleans: The Society of Naval Architects and Marine Engineers; 1992. p. 7C2-1 - 7C2-11.

[10] Bedi HS, Arora H, Bansal M. Microstructures, mechanical properties \& corrosion behavior of duplex 2209 in electroslag strip cladding over low carbon steel substrate: a review paper. International Journal of Engineering Research and Applications. 2015;5(3):72-73.

[11] Kahar SD, Baba Pai K. Corrosion behavior of electro-slag strip cladded weld overlays in different acid solutions. International Journal of Engineering Research and Applications. 2013;3(4):26202627.

[12] Kumar MK, Das S. A Review on different cladding techniques employed to resist corrosion. Journal of the Association of Engineers. 2016;86(1-2):51-63.

[13] Cavalcante NE, Andrade TC, Pinheiro PHM, Miranda HC, Motta MF, Aguiar WM. Estudo de procedimentos de soldagem MIG/ MAG para aplicação de revestimentos de liga de níquel Inconel
625 em aço estrutural ASTM A387 Gr.11. Soldagem \& Inspeção. 2016;21(1):70-82. http://dx.doi.org/10.1590/0104-9224/ SI2101.07.

[14] Petrzak P, Blicharski M, Dymek S, Solecka M. Electron microscopy investigation of Inconel 625 weld overlay on boiler steel. Solid State Phenomena. 2015;231:113-118. http://dx.doi.org/10.4028/ www.scientific.net/SSP.231.113.

[15] Alvarães CP, Madalena FCA, Araújo LS, Souza LFG, Jorge JCF. Propriedades de revestimentos de liga Inconel 625 obtidos pelos processos arame tubular e eletrodo revestido. In: Anais do $70^{\circ}$ Congresso Anual da ABM; 2015 Ago 17-21; Rio de Janeiro, Brasil. Rio de Janeiro: Associação Brasileira de Metalurgia e Materiais; 2015. p. 1-11.

[16] Gornikowska RM, Cieniek L, Blicharski M, Kusinski J. Microstructure and microsegregation of an Inconel 625 weld overlay produced on steel pipes by the cold metal transfer technique. Archives of Metallurgy and Materials. 2014;59(3):1081-1084.

[17] Farkade NV, Ravanan PN. Modification in weld overlay for productivity and corrosion resistance. International Journal of Science Technology \& Engineering. 2015;2(2):37-41.

[18] Ribeiro RA. Análise comparativa de uma junta API 5L X65 revestida com Inconel 625 pelos processos TIG automatizado e soldagem por explosão [dissertação de mestrado]. Rio de Janeiro: Universidade Estadual do Norte Fluminense; 2014.

[19] Du Pont JN, Lippold JC, Kiser SD. Welding metallurgy and weldability of nickel-based alloys. New Jersey: John Wiley \& Sons, Inc.; 2009.

[20] American Society of Mechanical Engineers. ASME Boiler and Pressure Vessel Code, Section IX: Qualification Standard for Welding, Brazing, and Fusing Procedures; Welders; Brazers; and Welding, Brazing, and Fusing Operators. New York: ASME; 2013.

[21] American Society for Testing and Materials. ASTM G48: standard test methods for pitting and crevice corrosion resistance of stainless steels and related alloys by use of ferric chloride solution. West Conshohocken: ASTM; 2009.

[22] Norsok Standard. NORSOK M-601: welding and inspection of piping. LysakerNorway: Norsok; 2004.

[23] Paschold R. Electroslag strip cladding for corrosion resistance. Svetsaren. 2001;56(2-3):62-67.

[24] Santos A, Possebon S, Martins FJS. Soldagem de tubulações revestidas utilizando metal de adição NiCrMo-3 (liga de níquel 625): aspectos metalúrgicos. In: Anais do $39^{\circ}$ Congresso Brasileiro de Soldagem; 2013 Nov 25-28; Curitiba, Brasil. Curitiba: Associação Brasileira de Soldagem; 2013. p. 1-14.

[25] Ferrari M. Qualificação de procedimento de soldagem para juntas de união em tubulações de API $5 \mathrm{~L}-\mathrm{X} 60$ revestidas internamente com liga de níquel 625. In: Anais do $37^{\circ}$ Congresso Brasileiro de Soldagem; 2011 Out 3-6; Natal, Brasil. Natal: Associação Brasileira de Soldagem; 2011. p. 1-10.

[26] Santos AX, Maciel TM, Santana RAC. Avaliação de revestimentos a base de Inconel 625 depositados através do processo de soldagem GMAW em aço API 5L X70 utilizando planejamento 
fatorial. Revista Brasileira de Aplicações de Vácuo. 2015;34(3):128140. http://dx.doi.org/10.17563/rbav.v34i3.995.

[27] Rutzinger $B$. Influence of the welding process to the dilution rate of weld overlays on unalloyed steel using the weld consumable ERNiCrMo-3 (Alloy 625). Biuletyn Instytutu Spawalnictwa. 2015;5:72-75.

[28] Madalena FCA, Jorge JCF, Souza LFG, Costa HRM, Cabral TB. Estudo de propriedades mecânicas e microestruturais do aço inoxidável superaustenítico AISI 904L utilizado como revestimento interno em vasos de pressão fabricados em aço carbono ASTM A-516 grau 70. In: Anais do $7^{\circ}$ Congresso Brasileiro de Engenharia de Fabricação; 2013 Maio 20-24; Penedo, Brasil. Penedo: Associação Brasileira de Engenharia e Ciências Mecânicas; 2013. p. 1-12.

[29] Cieslak MJ, Headley TJ, Romig AD, Kollie T. A melting and solidification study of alloy 625. Metallurgical Transactions. A, Physical Metallurgy and Materials Science. 1988;19A(9):23192331. http://dx.doi.org/10.1007/BF02645056.

[30] Du Pont JN, Banovic SW, Marder AR. Microstructural evolution and weldability of dissimilar welds between a super austenitic stainless steel and nickel-based alloys. Welding Journal. 2003;82(6):125-135.

[31] Di JX, Chen B. High chromium nickel base alloy weld deposited metal. Science and Technology of Welding and Joining. 2015;20(4):325-329. http://dx.doi.org/10.1179/1362171 $815 Y .0000000019$.

[32] Gornikowska MR, Blicharski M. Microsegregation and precipitates in Inconel 625 arc weld overlay coatings on boiler pipes. Arquives of Metallurgy and Materials. 2015;60(4):2599-2605. http:// dx.doi.org/10.1515/amm-2015-0420.

[33] Cieslak MJ, Knorovsky GA, Headley TJ, Roming AD Jr. The Solidification metallurgy of alloy 718 and other $\mathrm{Nb}$-containing superalloys. In: Proceedings of the International Symposium on the Metallurgy and applications of Superalloy 718; 1989 Jun 12-14; Pittsburgh, USA. Pittsburgh: The Minerals, Metals \& Materials Society; 1989. p. 59-68.
[34] Porter DA, Easterling KE. Phase transformations in metals and alloys. 2nd ed. London: Chapman \& Hall; 1997

[35] Cieslak MJ. The welding and solidification metallurgy of alloy 625. Welding Journal. 1991;70(2):49-56.

[36] Du Pont JN. Solidification of an alloy 625 weld overlay. Metallurgical and Materials Transactions. A, Physical Metallurgy and Materials Science. 1996;27A(11):3612-3620. http://dx.doi. org/10.1007/BF02595452.

[37] Xu F, Lv Y, Liu Y, Shu F, He P, Xu B Microstructural evolution and mechanical properties os Inconel 625 alloy during pulsed plasma are deposition process. Journal of Materials Science and Technology. 2013;29(5):480-488. http://dx.doi.org/10.1016/j. jmst.2013.02.010.

[38] Solecka M, Petrzakb P, Radziszewska A. The microstructure of weld overlay Ni-base alloy deposited on carbon steel by CMT method. Solid State Phenomena. 2015;231:119-124. http:// dx.doi.org/10.4028/www.scientific.net/SSP.231.119.

[39] Nace International. NACE MR0175: Petroleum and natural gas industries - Materials for use in H2S-containing environments in oil and gas production; Part 2: Cracking-resistant carbon and low-alloy steels, and the use of cast irons. Houston: Nace International; 2009.

[40] Shen RR, Zhou Z, Liu P, Chai G. Effects of PWHT on the microstructure and mechanical properties of ERNiCrFe-7 all-weld metal. Welding in the World. 2015;59(3):317-323. http://dx.doi. org/10.1007/s40194-014-0201-4.

[41] Suave LM, Cornier J, Villechaise P, Soula A, Hervier Z, Bertheaud $D$, et al. Microstructural evolutions during thermal aging of alloy 625: impact of temperature and forming process. Metallurgical and Materials Transactions. A, Physical Metallurgy and Materials Science. 2014;45A(7):2963-2982. http://dx.doi.org/10.1007/ s11661-014-2256-7.

[42] Suave LM, Cornier J, Villechaise P, Soula A, Hervier Z, Bertheaud $D$, et al. Impact of microstructural evolutions during thermal aging of alloy 625 on its monotonic mechanical properties. MATEC Web of Conference. 2014;14. http://dx.doi.org/10.1051/ matecconf/20141421001. 